\title{
Auscultation on the Ambulance: A Case Study of Adaptive Filter Application for the Safety of Detecting Lung Sound on the Ambulance
}

\author{
Bing-Yuh Lu ${ }^{1,2}$, Meng-Lun Hsueh ${ }^{3}$, Hung-Wen Hung ${ }^{1}$, Hai-Wu Lee ${ }^{4}$, Jun-Xian Yu ${ }^{5}$ and Shui-Ken Wei ${ }^{1}$ \\ 1. Department of Electronic Engineering, Tungnan University, New Taipei City 22202, Taiwan, Republic of China \\ 2. Department of Business Administration, National Taipei University of Business, Taipei 100, Taiwan, Republic of China \\ 3. Department of Electronic Engineering, HwaHsa University of Technology, New Taipei City 236, Taiwan, Republic of China \\ 4. Department of Electronic Engineering, National Taipei University of Technology, Taipei 106, Taiwan, Republic of China \\ 5. Department of Electronic Engineering, Xiehe Youde Senior High School, Taipei 106, Taiwan, Republic of China
}

\begin{abstract}
This study investigates the priority of the auscultation on the ambulance. On the point of physics view, the electrical noise cancellation is simpler than mechanical one. Therefore, the difficulties of the measurement the physiological sounds are more than that of physiological electrical signals. Therefore, the medical doctors might practically consider the priority of the emergent measurement on the ambulance. This study presented the application of the traditional adaptive filter to reduce the interference of the sound of sirens (audible warnings) of the ambulance. The auscultation of respiration on the ambulance is usually different from that in clinic room. The environmental noise is much larger on the ambulance, especially, the sound from audible warnings. However, this study simulated the filtering effects of the adaptive filter for the lung sound auscultation on the ambulance. The results showed that the harmonics of the audible warning were completely cancelled, and the component of fundamental frequency of that was reduced from $-30 \mathrm{~dB}$ to $-60 \mathrm{~dB}$. We proposed a simple time-domain method to enhance the respiration sound to support the higher quality auscultation information for medical doctors, and aimed at concurring the barriers to elevate the priority of the measurement on ambulance for meeting with the necessaries of medical diagnosis.
\end{abstract}

Key words: Acoustic signal, lung sound, adaptive filter, distant healthcare, priority.

\section{Introduction}

When a patient is on the ambulance, it is very possible that the medical staffs need to collect the physiological signals for monitoring and ensuring the safety of the patient [1]. Therefore, the previous study has investigated a valuable research to sort the priority of the signals to be transferred from the ambulance. The stethoscope was listed in the 15 items in the table in Protogerakisel al.'s study which was considered by the practical factors of the different necessary signals to be transmitted from the emergency site and their priority with a classification into continuous or intermittent transmission and the priority for

Corresponding author: Bing-Yuh Lu, Ph.D., research field: signal processing. transmission [2].

Rene Laennec invented the first stethoscope in $\mathbf{1 8 1 6}$ [1]. Auscultation is listening to the internal sounds of the body. The heart, lungs, and gastrointestinal tract are frequently measured by auscultation. Therefore, auscultation can be used to detect the physiological sounds produced by the circulatory, respiratory, and digestive systems. Doctors generally apply standard auscultation procedures for diagnoses.

Real-time distant healthcare is crucial in examining the heart and lung sounds of patients. Respiratory sounds occur both of the durations of inspiration and expiration. The reasons of the respiration sounds occur as the air moves in and out of the chest when a person breathes normally. Therefore, the auscultation of 

for the Safety of Detecting Lung Sound on the Ambulance

normal breathing sounds and abnormal or adventitious sounds can be detected by the stethoscopes [3, 4]. However, the interference of the sound of sirens of the ambulance is always influent to the auscultation. Therefore, a speedy algorithm to process the cancellation of the sound of sirens is necessary to the auscultation on ambulance or sent the respiration to the distant emergency center in the hospital. Therefore, we proposed an algorithm of adaptive filter, and simulated the performance of the algorithm as the preparation before implementing the auscultation system on the ambulance.

Furthermore, the critical environment conditions of diagnosis and monitoring of patients in a variety of emergency and military medicine situations. Those include the environments in field hospitals, in high ambient noise environments such as engine rooms, in vehicles such as ambulances, fixed and rotary wing aircraft, or in hyperbaric chamber devices [5]. Therefore, we have to emphasize the importance of the auscultations in the special situations.

This article presents the key perspectives on auscultation in the section of introduction; the adaptive filter design in the section of method; the main noise reduction in the section of results; and overview examinations of the auscultation on the ambulance in the sections of discussion, and conclusions.

\section{Method}

Fig. 1 presented the lung sound auscultation on the ambulance, where (a) was the acoustic signal in time domain, and (b) was the spectrogram. In the spectrogram, the fundamental tone, its harmonics of the siren and its echoes are higher than $750 \mathrm{~Hz}$. Therefore, designing a low-pass filter to cancel the sound from siren, and then the output signal of the filter became the desired signal of the adaptive filter.

The adaptive filter algorithm is a traditional method which based on the computation of statistics and weightings to solve the noise cancellation. The optimization of the cost function is the key to the LMS (least mean squares) algorithms [6]. One of the advantages of the LMS algorithm is that it can be employed without complex matrix operations.

The computation procedures of standard LMS adaptive filter are presented as follows:

Step 1: Calculates the output signal $\mathrm{y}[\mathrm{n}]$ by using the following eauation:

$$
\mathrm{y}[\mathrm{n}]=\widehat{w}^{H}[n-1] u[n]
$$

Step 2: Calculates the error signal $e[n]$ by using the following equation:

$$
e[n]=d[n]-y[n]
$$

Step 3: Updates the filter coefficients by using the following equation:
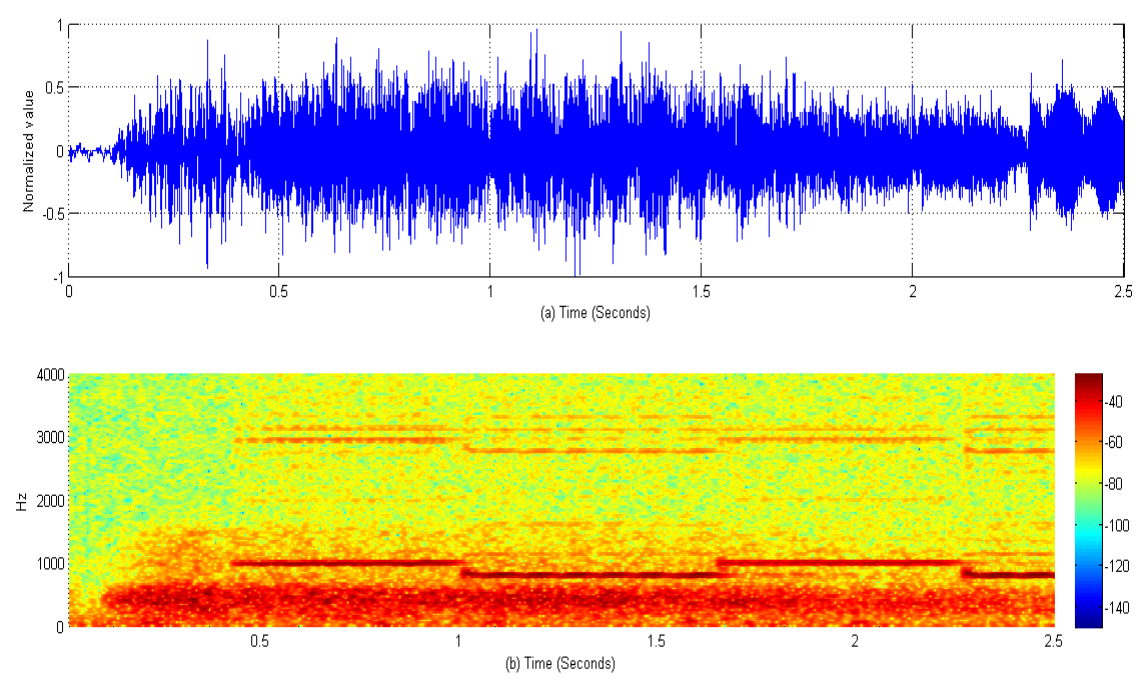

Fig. 1 Lung sound auscultation on the ambulance (original sound A), where (a) was the acoustic signal in time domain, and (b) was the spectrogram. 


$$
\widehat{w}[n+1]=\widehat{w}[n]+\frac{u[n]}{\alpha u^{H}[n] u[n]} \mu e^{*}[n]
$$

where $n$ denotes for the current algorithm iteration; $u[n]$ denotes for the buffered input samples at step $n$; $\widehat{w}[n]$ represents for thatthe vector of filter-tap estimates at step $\mathrm{n} ; \mathrm{N}$ is the number of the weighting tap; $\alpha$ is a coefficient; $y[n]$ represents for the filtered output at step; $e[n]$ is for the estimation error at step $n$; $d[n]$ is for the desired response at step $n$; and $\mu$ is the adaptation step size.

Observing the spectrogram in Fig. 1, the frequency components of the desired signal are all distributed under $750 \mathrm{~Hz}$. Therefore, the respiration sound with the noise of the siren on the ambulance input a low-pass filter, and the output of the filter was defined as $\mathrm{d}[\mathrm{n}]$. The cutoff frequency was at 640 to $720 \mathrm{~Hz}$ with the attenuation of -1 to $-60 \mathrm{~dB}$. Therefore, Fig. 2 presented the functional block diagram of the filter design. $x[n]$ denotes for the input which was recorded on the ambulance. L.P.F is the transfer function of the low-pass filter. A.F.A. is the function of the adaptive filter, and $y[n]$ is the output of the adaptive filter.

Many researchers of the heart and lung sound analysis employed the technology of spectrogram analysis to explore the characteristics of the heart and lung sound. The spectrogram discloses the components of signal in both time and frequency domains. Therefore, it is usually to present the respiration signals in spectrogram. The element at the time $\tau$ in a spectrogram is defined as Ref. [7]:

$$
\left|P_{\tau}(j \omega)\right|^{2}=\left|\int_{-\infty}^{\infty} p(t) \emptyset_{\tau, \omega}(t) d t\right|^{2}
$$

$p(t)$ is the signal in the time domain, $\emptyset_{\tau, \omega}(t)$ is the complex basis function, and $\left|\mathrm{P}_{\tau}(\mathrm{j} \omega)\right|^{2}$ is the power distribution in the frequency domain at the time $\tau$. This form of the Fourier transform, also known as the STFT (short-time Fourier transform), has numerous applications in speech, sonar, and radar processing. The spectrogram of a sequence is the magnitude of the time-dependent Fourier transform versus time [8].

\section{Results}

The parameters in the adaptive filter are much influent to the output. Therefore, most of the relative researches are usually discussed the convergence of the output. In this study, the parameters of $\alpha, \mathrm{N}$ and computing time $\left(\mathrm{T}_{\mathrm{c}}\right)$ have been examined. The arrangement of Table 1 is to detect the better order of $\alpha$. Examining the spectrograms from Fig. 3 to Fig. 6, we found the spectra of Fig. 3 and Fig. 4 have been reduced in almost the same CPU time.

Based on the detection of the better $\alpha$ in Table 1, it is reasonable to set $\alpha=0.05$ for the further searching of the better parameters. In Figs. 5 and 6, the less orders of $\alpha$ did not cancel the noises of siren well. The reason was that the less orders caused the slower convergent speed. Consequently, Table 2 has been designed for the searching of the better $\mathrm{N}$.

According to the detection of the better $\mathrm{N}$ in Table 2, we found that $\mathrm{N}=64$ when $\alpha=0.05$ were the better parameters for the siren noise cancellation. The spectrograms in Figs. 10 and 11 show the complete cancellation of sound of siren, but the clear frequency boundary discloses the overweighting in the adaptive filter algorithm. Therefore, $\mathrm{N}=64$ is selected to be the better value in the algorithm.

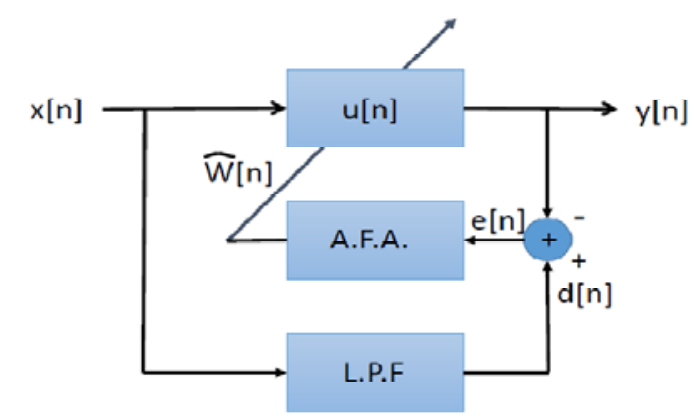

Fig. 2 The block diagram of the adaptive filter. (A.F.A: adaptive filter algorithm; L.P.F: low-pass filter).

Table 1 Find the better order of $\alpha$.

\begin{tabular}{lccll}
\hline$\#$ & $\alpha$ & $\mathrm{N}$ & $\mathrm{T}_{\mathrm{c}}$ & Figure \# \\
\hline 1 & 0.1 & 64 & 2515.4 & Fig. 3 \\
2 & 0.01 & 64 & 2620.2 & Fig. 4 \\
3 & 0.001 & 64 & 2642.5 & Fig. 5 \\
4 & 0.0001 & 64 & 2668.7 & Fig. 6 \\
\hline
\end{tabular}



for the Safety of Detecting Lung Sound on the Ambulance
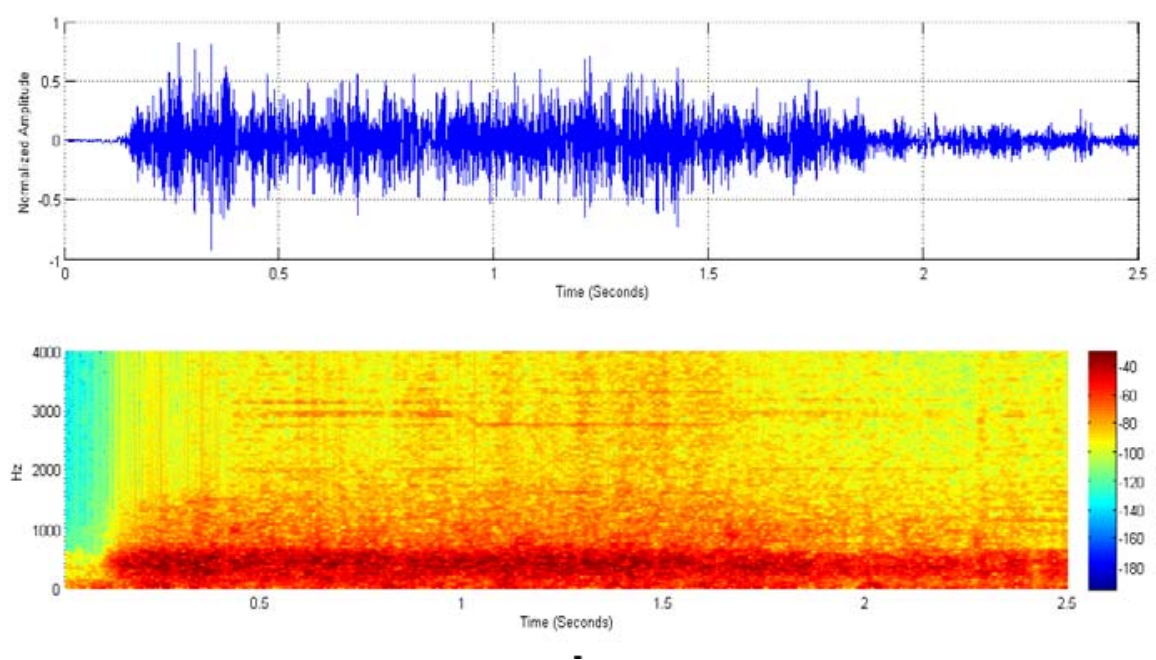

Fig. $3 \alpha=0.1$.
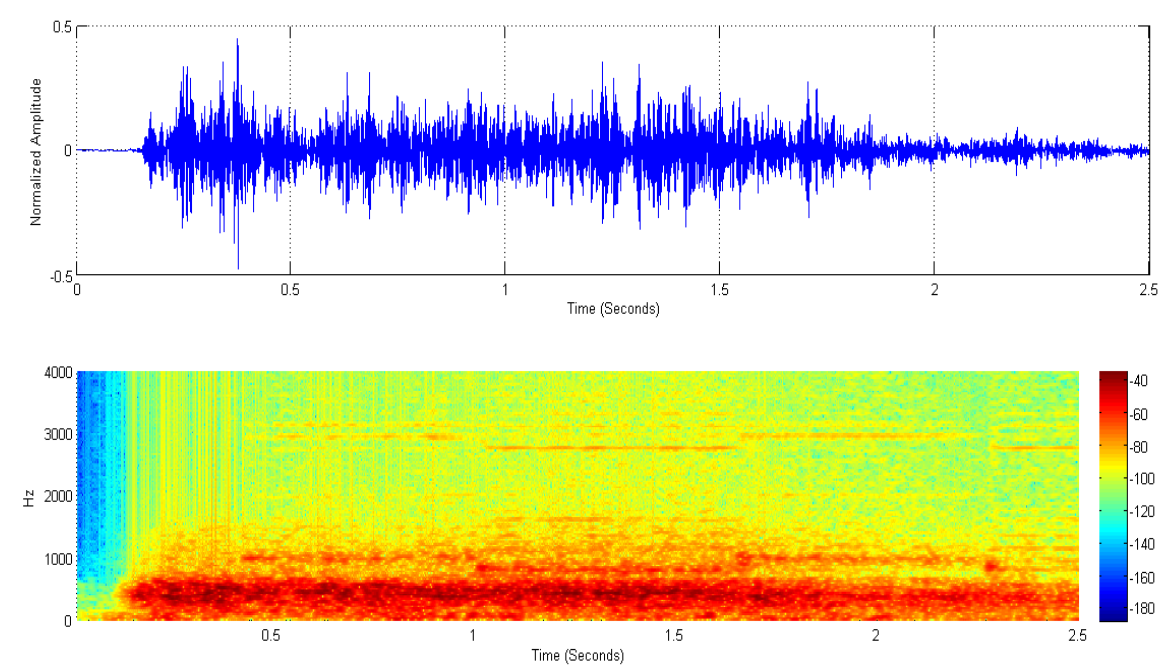

Fig. $4 \alpha=0.01$.
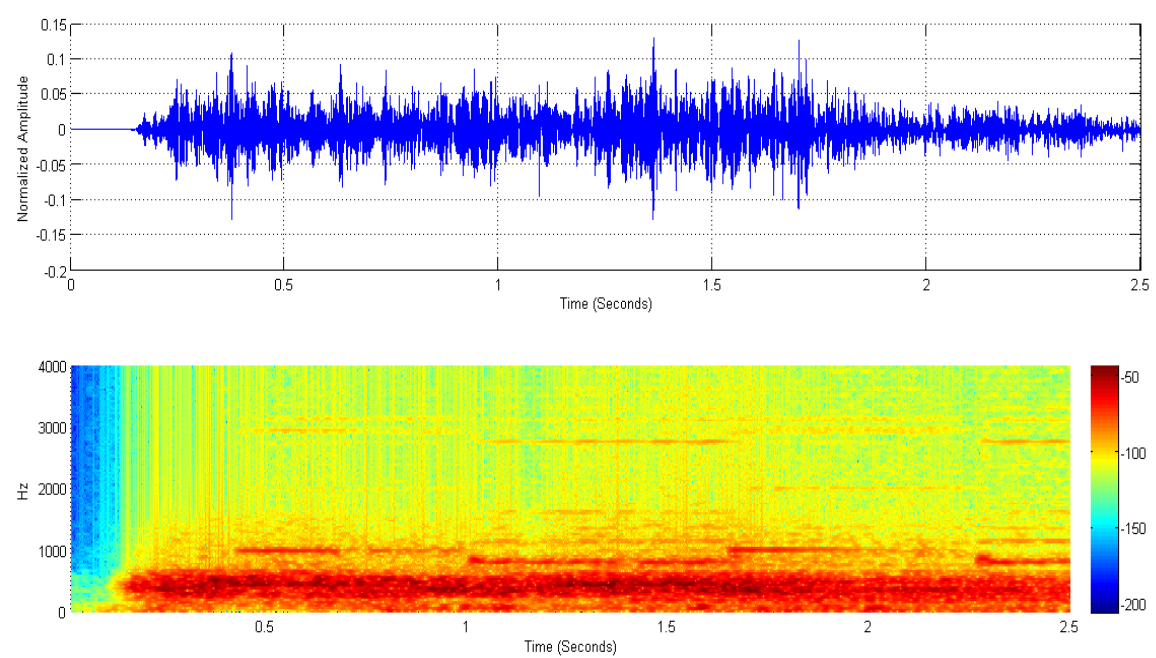

Fig. $5 \alpha=0.001$. 
Auscultation on the Ambulance: A Case Study of Adaptive Filter Application

for the Safety of Detecting Lung Sound on the Ambulance
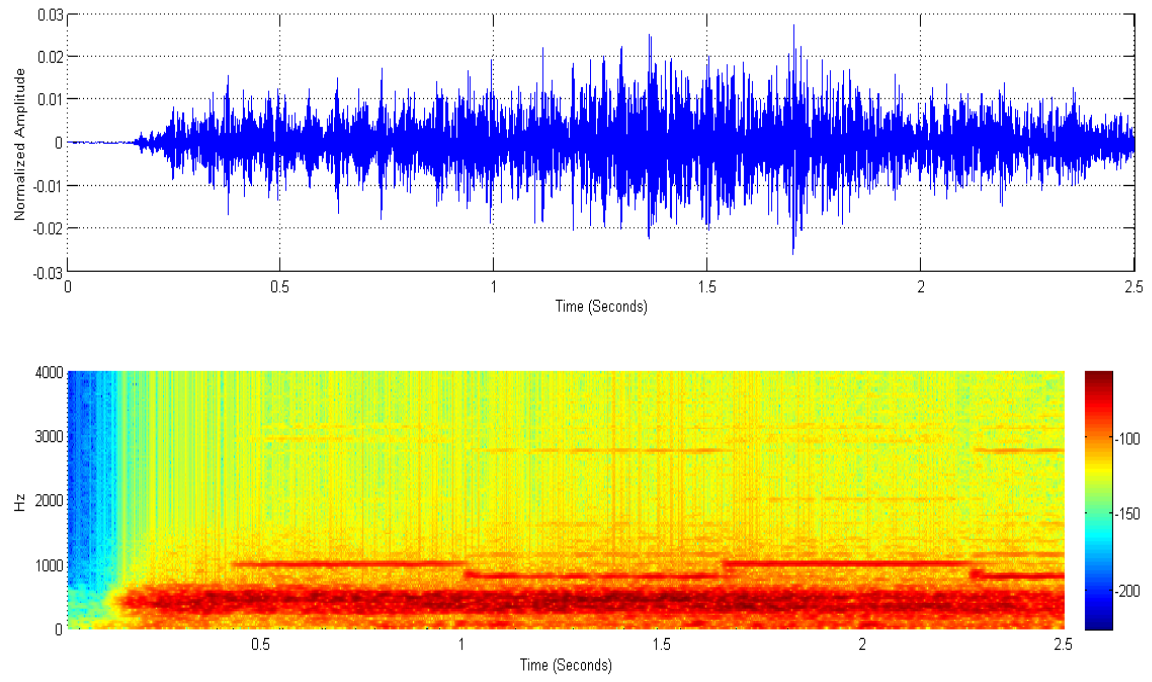

Fig. $6 \alpha=0.0001$.
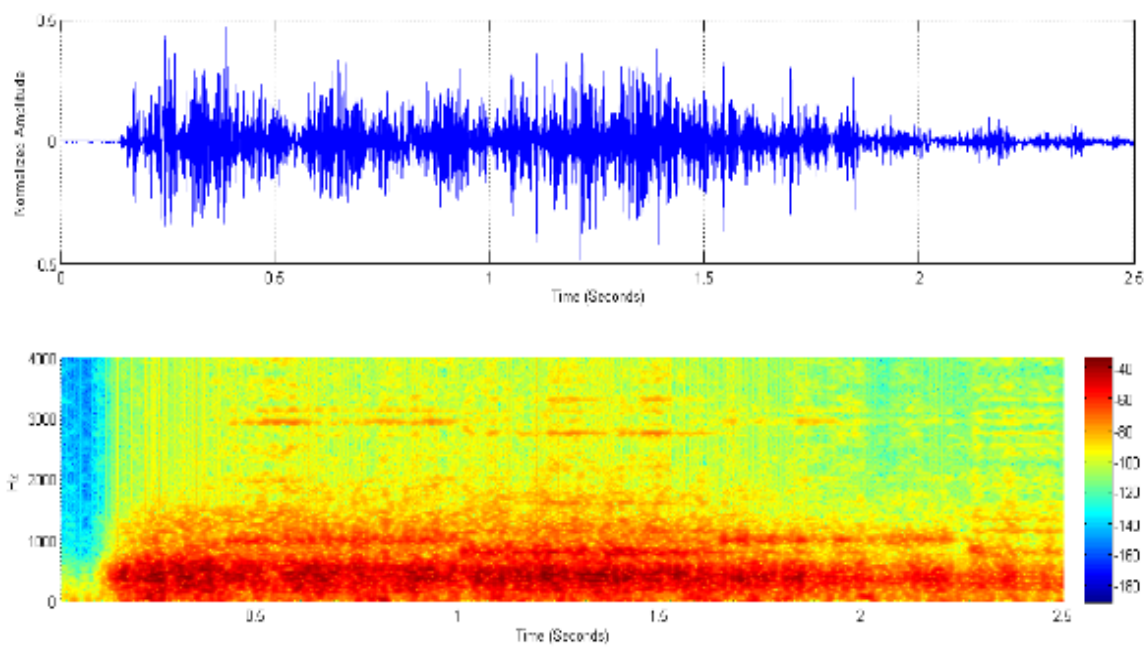

Fig. $7 \quad \mathrm{~N}=16$.
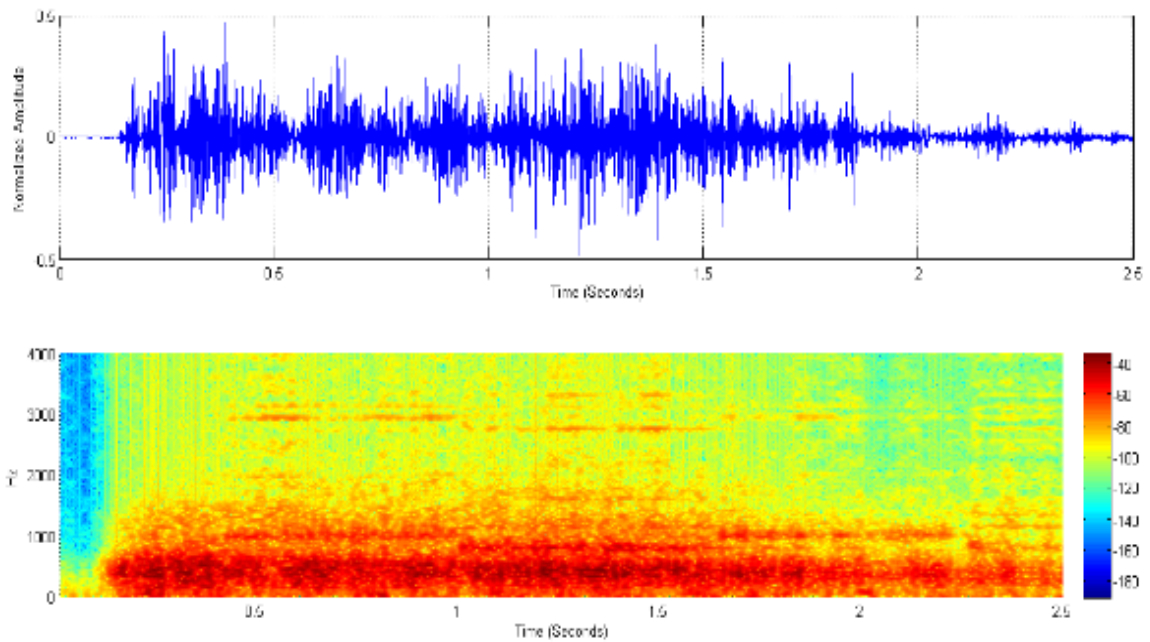

Fig. $8 \quad \mathrm{~N}=32$. 

for the Safety of Detecting Lung Sound on the Ambulance
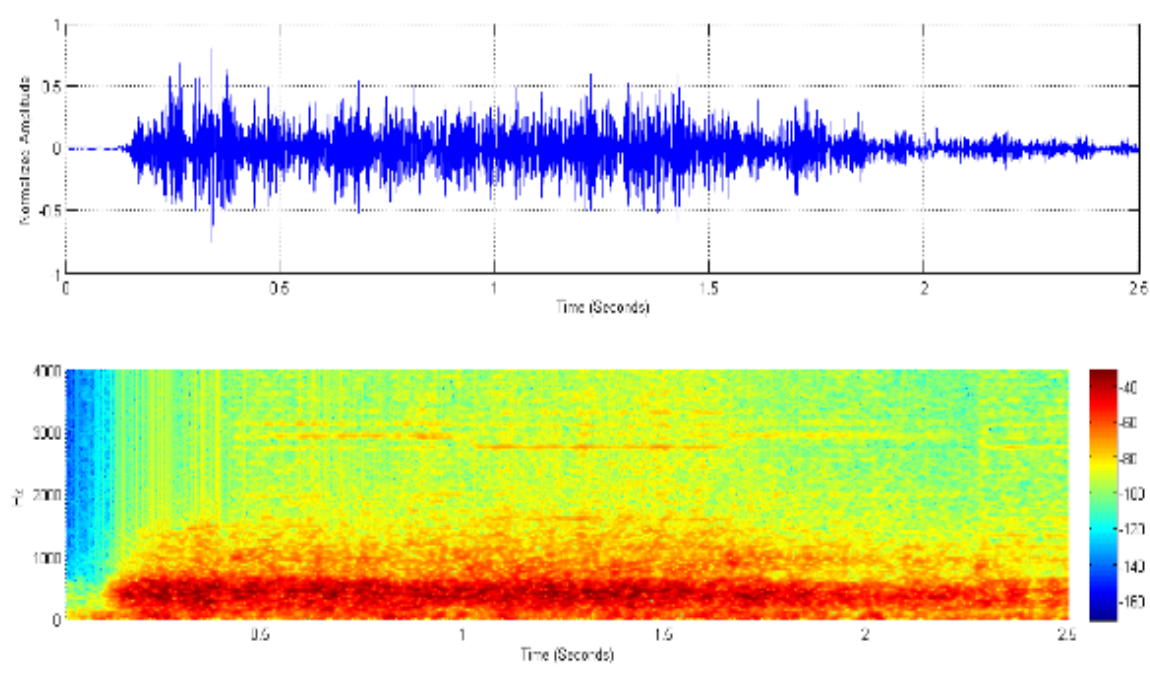

Fig. $9 \quad \mathrm{~N}=64$.
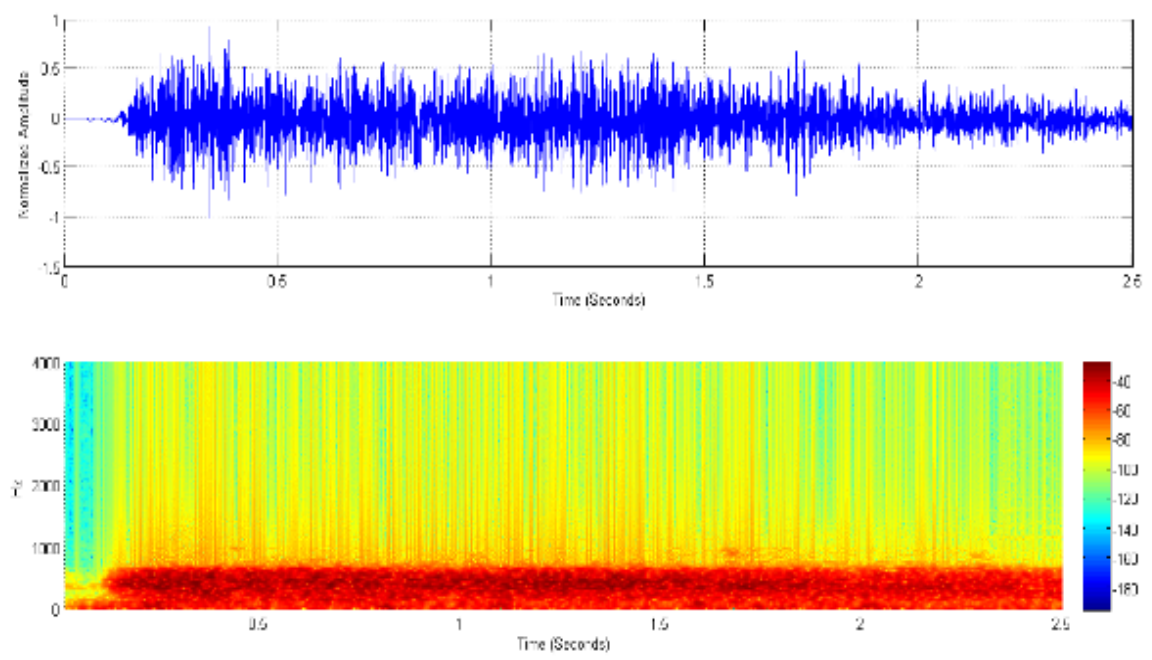

Fig. $10 \quad N=128$.
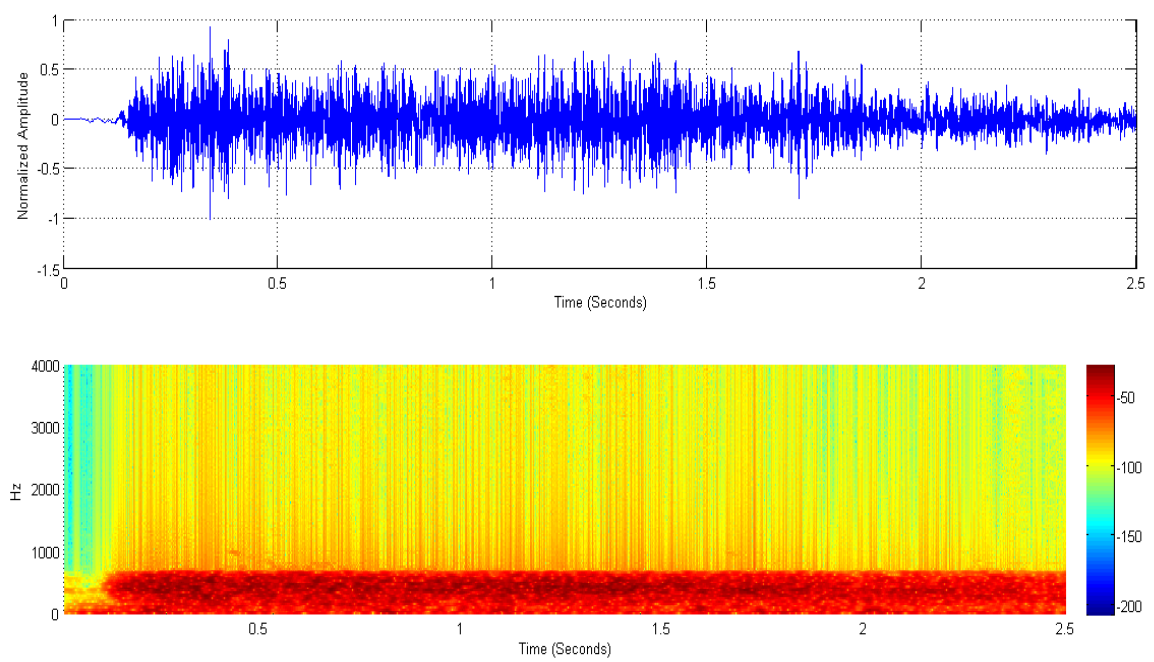

Fig. $11 \quad N=256$. 
Table 2 Searching of the better $\mathbf{N}$.

\begin{tabular}{|c|c|c|c|c|}
\hline \# & $\alpha$ & $\mathrm{N}$ & $\mathrm{T}_{\mathrm{c}}$ & Figure \# \\
\hline 1 & 0.05 & 16 & 2750.7 & Fig. 7 \\
\hline 2 & 0.05 & 32 & 2773.0 & Fig. 8 \\
\hline 3 & 0.05 & 64 & 2799.4 & Fig. 9 \\
\hline 4 & 0.05 & 128 & 2866.2 & Fig. 10 \\
\hline 5 & 0.05 & 256 & 2888.9 & Fig. 11 \\
\hline 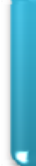 & $\begin{array}{l}\text { Adaptive } \\
\text { filter }\end{array}$ & & $\begin{array}{l}\text { 2nd order } \\
\text { low-pass } \\
\text { Butterworth } \\
\text { filter }\end{array}$ & $\begin{array}{l}\text { Sound for } \\
\text { auscultation }\end{array}$ \\
\hline
\end{tabular}

Fig. 12 The functional block diagram for improving the auscultation.

\section{Discussion}

Compared with the modern artificial intelligent (AI) algorithms such as fuzzy logic, neuron network (ANN), and so on, the adaptive filter algorithm was presented much traditional analytic and simple. Therefore, we proposed it as a traditional method to solve the noise of siren on the ambulance to explain the feasibility of the detecting a clear lung sound was very clear, but did not perform well in the previous auscultation on the ambulances.

In our experiences, auscultation is usually the 1 st priority process of the diagnosis in the internal medicine, and relevant departments. The diagnosis of the medical doctors of internal medicine traditionally begins at the auscultation, because the heart sounds or lung sounds support the basic classification information of the physiological signs. Therefore, the serious noise influence had been needing to be concurred. Therefore, the value of our study is disclosed.

To achieve the clearer sounds of auscultations, the lower order of low-pass filters are frequently inserted in the stethoscopes to reduce the higher frequency environmental noises. Therefore, we attempted to improve the sound in Fig. $9(\mathrm{~N}=64$, and $\alpha=0.05)$ by passing through a low-pass Butterworth filter. The functional block diagram was presented in Fig. 12. The output of the aforementioned adaptive filter input the second order low-pass Butterworth filter which slightly reduced the higher frequency sounds. The output of the adaptive filter at the condition of $\mathrm{N}=64$, and $\alpha=0.05$ (Fig. 9) has been processed by a second order low-pass Butterworth filter presented in Fig. 13. We have to point out that in the end of the sound signal in the original Fig. 1 showed the harmonic sound of siren. Through the processing in Fig. 12, the results disclosed the separation of the respiration and siren sounds.

For the serious examination of the aforementioned method that was proposed in this study, another original auscultation sound (original sound B, Fig. 14) was repeated all the processes as the functional block diagram in Fig. 12 to test the performance of the filter design. The result of the processed original sound B (Fig. 14) was presented in Fig. 15 which clearly displayed some segments of the breath sounds in the time domain. The proposed adaptive filter algorithm was proven to be a feasible method for the auscultation on the ambulances. The results showed that the harmonics of the audible warning were completely cancelled, and the component of fundamental frequency of that was reduced from -30 $\mathrm{dB}$ to $-60 \mathrm{~dB}$.

$\mathrm{Lu}$ and $\mathrm{Wu}[9,10]$ have proposed that relatively simple trial of a potential method for transmitting audio signal derived from auscultation. Contemporary smart phones are widely used because numerous new applications (APPs) are available for the new generation of smart phones. Based on new wireless communication technologies, which include Bluetooth ${ }^{\circledR}$ technology, the modern mobile communication systems, and modern electronic stethoscopes, we developed a real-time mobile-based auscultation (RMA) that can be applied in distant health care to improve the convenience of performing real-time auscultation on patients. Consequently, the solutions of auscultation on ambulance or distant auscultation in the emergency center in hospital has been found to realize the system in the near future. 

for the Safety of Detecting Lung Sound on the Ambulance
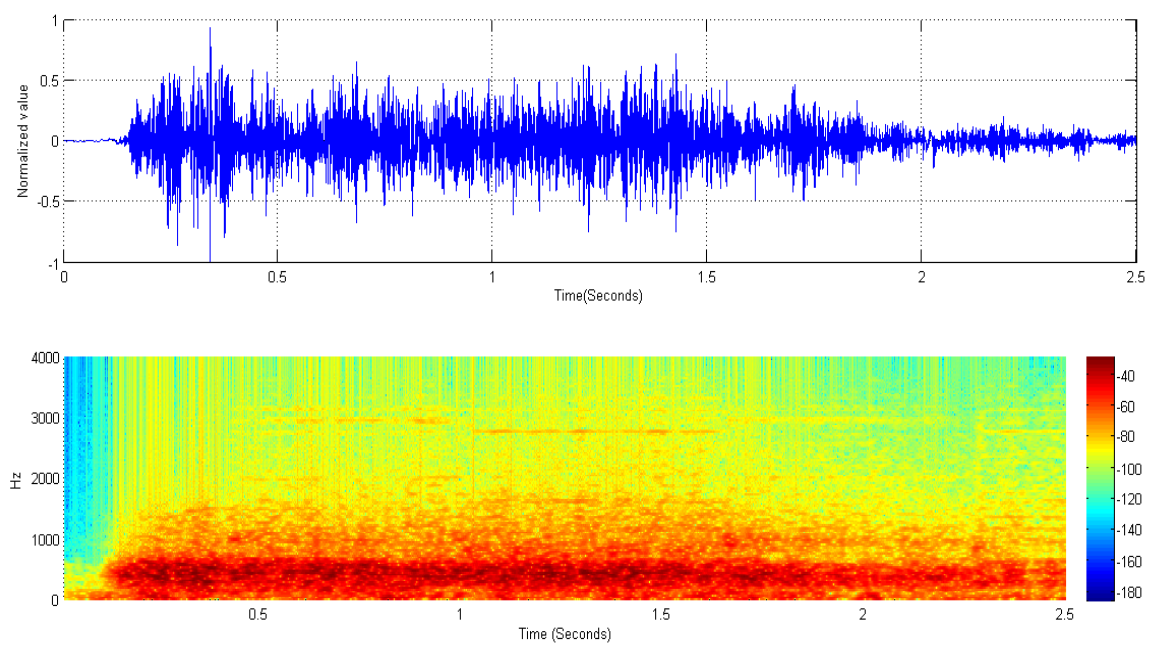

Fig. 13 The output of the adaptive filter at the condition of $N=64$, and $\alpha=0.05$ (Fig. 9) has been processed by a second order low-pass Butterworth filter.
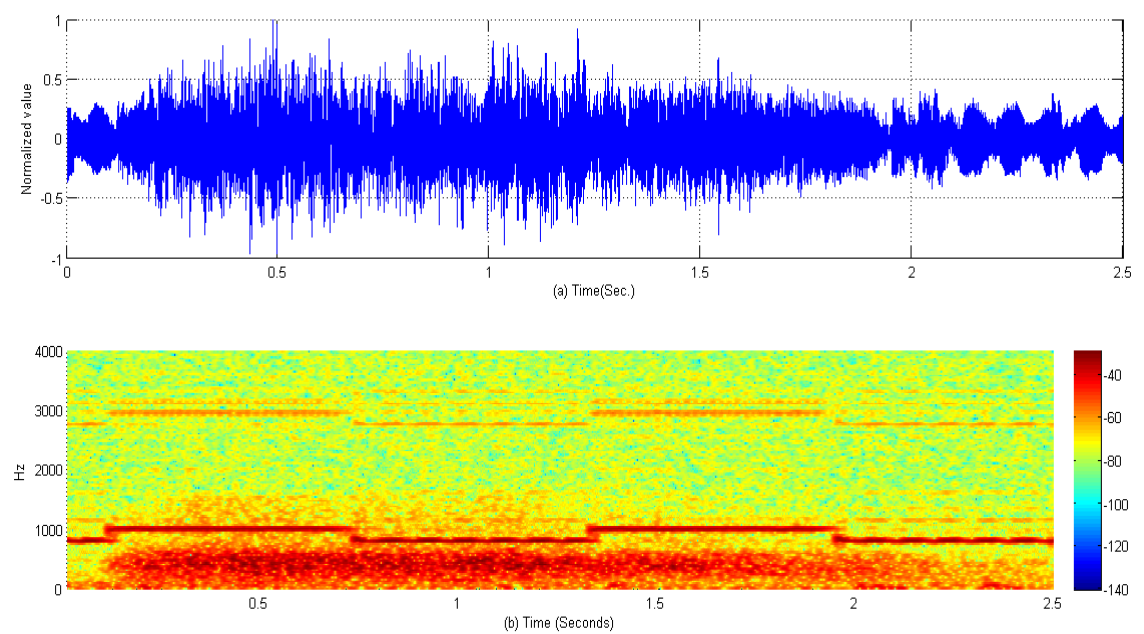

Fig. 14 Another original auscultation sound (original sound B) on the ambulance.
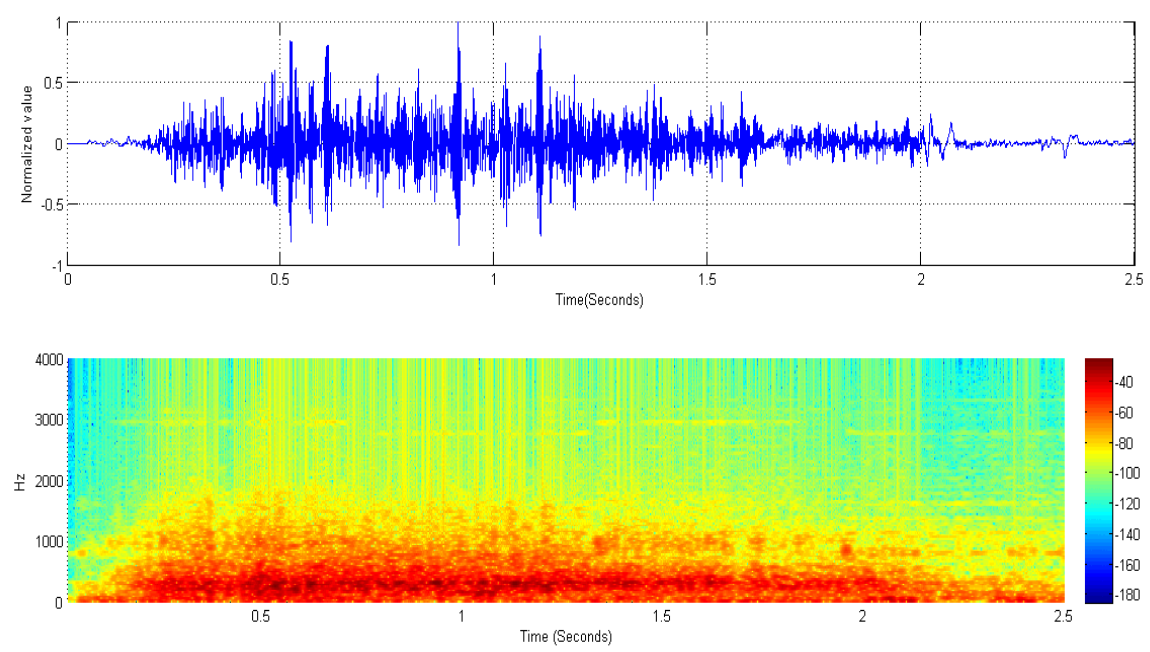

Fig. 15 The processed original sound $B$ on the ambulance. 


\section{Conclusions}

The adaptive filter is a signal processing algorithm in time domain. Therefore, it is very potential to develop to be a real-time noise cancellation device for the auscultation on the ambulances. The proposed adaptive filter algorithm was proven to be a feasible method for the auscultation on the ambulances. The results showed that the harmonics of the audible warning were completely cancelled, and the component of fundamental frequency of that was reduced from $-30 \mathrm{~dB}$ to $-60 \mathrm{~dB}$. $\mathrm{Lu}$ and $\mathrm{Wu}[9]$ have proposed that relatively simple trial of a potential method for transmitting audio signal derived from auscultation. Consequently, the solutions of auscultation on ambulance or distant auscultation in the emergency center in hospital have been found to realize the system in the near future.

Furthermore, we proposed it as a traditional method to solve the noise of siren on the ambulance to explain the feasibility of the detecting a clear lung sound was very clear, but did not perform well in the previous auscultation on the ambulances [11, 12]. The barriers of the clear auscultation sounds on the ambulances might change the priority of the medical doctors to present their necessity for precise diagnoses. However, we employed the proposed traditional adaptive filter algorithm to encourage the solutions of the auscultation on the ambulances.

Finally, the critical environment conditions of diagnosis and monitoring of patients are in a variety of emergency medicine situations. Therefore, we have to emphasize the importance of the auscultations in the special situations.

\section{Acknowledgements}

The authors thank Professor Gwo-Ching Chang who is at the Department of Information Engineering, I-Shou University, Kaohsiung City, Taiwan, Republic of China provided the sound data to us. Furthermore, we thank for the supports from the project numbers of MOST 103-2221-E-236-001, and MOST 103-2627-E-002-005, Ministry of Science and Technology, Taiwan, Republic of China.

\section{References}

[1] Geddes, L. A. 2005. "Birth of the Stethoscope." IEEE Engineering in Medicine and Biology Magazine, Jan./Feb.: 84-6.

[2] Protogerakis, M., Gramatke, A., and Henning, K. 2009. "A Telematic Support System for Emergency Medical Services." In Proceedings of 3rd IEEE International Conference on Bioinformatics and Biomedical Engineering (ICBBE), 1-4.

[3] Liang, H., Lukkarinen, S., and Hartimo, I. 1997. "Heart Sound Segmentation Algorithm Based on Heart Sound Envelolgram." Computers \& Cardiology 24: 105-8.

[4] Chang, G. C., and Lai, Y. F. 2010. "Performance Evaluation and Enhancement of Lung Sound Recognition System in Two Real Noisy Environments." Computer Methods and Programs in Biomedicine 97(2): 141-50.

[5] Naval Submarine Medical Research Laboratory. 2000. Noise Reduction Stethoscope for United States Navy Application. NSMRL Report 1214.

[6] Hayes, M. H. 1996. Statistical Digital Signal Processing and Modeling. New York: Wiley.

[7] Ludeman, L. C. 2003. Random Processes Filtering, Estimation, and Detection, New York: Wiley.

[8] Emmanouilidou, D., McCollum, E. D., Park, D. E., and Elhilali, M. 2015. "Adaptive Noise Suppression of Pediatric Lung Auscultations with Real Applications to Noisy Clinical Settings in Developing Countries." IEEE Transactions on Biomedical Engineering 62 (9): 2279-88.

[9] Lu, B. Y., and Wu, H. D. 2015. "Auscultation Using Modern Mobile Communication." Acoustics Australia 43: 303-9.

[10] Lu, B. Y., and Sing, S. S., and Wu, H. D., Hsu, L. Y., Lai, J. S. 2015. "The Feasibility Study of Mobile-to-Mobile Communication for Auscultation of Heart Sound and Lung Sound." In Proceedings of 17th IEEE International Conference on Advanced Communication Technology, 387-93.

[11] Howard, C. Q., Maddern, A. J., and Privopoulos, E. P. 2011. "Acoustic Characteristics for Effective Ambulance Sirens." Acoustics Australia 39: 43-53.

[12] Haykin, S., and Van Veen, B. 1998. Signals and Systems. New York: Wiley. 